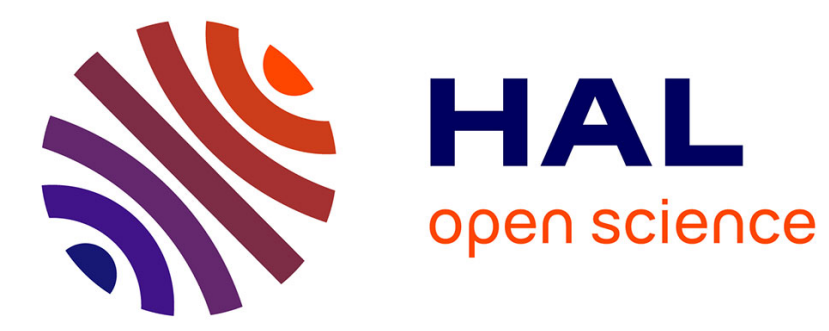

\title{
Role of the Website Structure in the Diversity of Browsing Behaviors
}

Pedro Ramaciotti Morales, Lionel Tabourier, Sylvain Ung, Christophe Prieur

\section{To cite this version:}

Pedro Ramaciotti Morales, Lionel Tabourier, Sylvain Ung, Christophe Prieur. Role of the Website Structure in the Diversity of Browsing Behaviors. 30th ACM Conference on Hypertext and Social Media, Sep 2019, Hof, Germany. pp.133-142, 10.1145/3342220.3343648 . hal-02422544

\section{HAL Id: hal-02422544 https://hal.science/hal-02422544}

Submitted on 22 Dec 2019

HAL is a multi-disciplinary open access archive for the deposit and dissemination of scientific research documents, whether they are published or not. The documents may come from teaching and research institutions in France or abroad, or from public or private research centers.
L'archive ouverte pluridisciplinaire HAL, est destinée au dépôt et à la diffusion de documents scientifiques de niveau recherche, publiés ou non, émanant des établissements d'enseignement et de recherche français ou étrangers, des laboratoires publics ou privés. 


\title{
Role of the Website Structure in the Diversity of Browsing Behaviors
}

\author{
Pedro Ramaciotti Morales ${ }^{1}$, Lionel Tabourier ${ }^{1}$, Sylvain Ung ${ }^{1}$, \\ and Christophe Prieur ${ }^{2}$ \\ ${ }^{1}$ Sorbonne Université, CNRS, LIP6, F-75005 Paris, France \\ ${ }^{2}$ I3, CNRS, Telecom Paris, Paris, France
}

\begin{abstract}
The quantitative measurement of the diversity of information consumption has emerged as a prominent tool in the examination of relevant phenomena such as filter bubbles. This paper proposes an analysis of the diversity of the navigation of users inside a website through the analysis of server log files. The methodology, guided and illustrated by a case study, but easily applicable to other cases, establishes relations between types of users' behavior, site structure, and diversity of web browsing. Using the navigation paths of sessions reconstructed from the log file, the proposed methodology offers three main insights: 1) it reveals diversification patterns associated with the page network structure, 2) it relates human browsing characteristics (such as multitabbing or click frequency) with the degree of diversity, and 3) it helps identifying diversification patterns specific to subsets of users. These results are in turn useful in the analysis of recommender systems and in the design of websites when there are diversity-related goals or constrains.
\end{abstract}

\section{Introduction}

In many areas such as life sciences, economics, finance, public policy, information theory, media studies, social sciences, and opinion dynamics, diversity 
refers to a property of a set of elements of interest, capable of revealing and quantifying notions such as variety, balance, and disparity [Sti07].

Much research has been conducted to characterize the diversity of information consumption in social media and news outlets. On the other hand there has been, for nearly two decades, a large amount of research dedicated to studying the browsing behavior of web users. Nonetheless, fewer studies have been devoted to the diversity of browsing behavior of users within a website. When reconstructed using transaction logs such as web server logs, navigation paths offer an interesting object of study. These allow for the investigation of the relationship between the page network structure and characteristics of human behavior when browsing. However, to relate this kind of analysis with the issue of diversity, one needs to get access to web server logs with both annotation for the contents over which to measure diversity, and annotated roles of webpages within the structure of a website.

The work presented in this article aims at exploring the question of how does the structure of a website influence the diversity of content explored by users. For this purpose, we use a web server log with the annotations of both the topic and the functional role (e.g. menu pages, forums, articles, ...) of the pages requested. Our approach is indeed illustrated on an original dataset, containing three weeks of web browsing on Melty, a prominent French information and entertainment website targeting young adults. Studies of navigation logs often focus on major web platforms, such as Facebook, Twitter or Wikipedia. Here, we have access to the logs of a site which can be described in terms of traffic as second-tier (a few million visits per week). Its structure and contents are quite typical of a category of websites which altogether represents a large part of the traffic on the web. We explore the patterns by which users are able to browse various topics and describe the relation between the functional role of a page, and the diversity of consumed information.

The main contribution of this article is a method to analyze the diversity of information consumption in browsing. We divide sessions into subgroups and aim at identifying different types of behaviors among users. From there, we define and measure patterns related to diversification processes and suggest explanations of how diversity consumption is affected by the site structure in our specific case study. However, the method can be applied to any case where it is possible to label pages based on their functional role, and also on the topic with which they deal. Such a situation is usual in e-commerce 
sites, social networks, or media outlets to name a few. We also analyze the temporal dynamics of diversity consumption, and its relation with bubblelike phenomena.

This article is organized in five sections. We first present related works on the two main topics discussed in this study: the measurement of diversity and the analysis of web logs. Then, we describe the dataset used, how it is preprocessed and how diversity will be defined and computed in the rest of the study. Next, we use this notion of diversity to explore the aggregated consumption of all users and motivate the analysis of the relation between the role and the content of pages. As we are looking for a natural way to classify browsing behaviors, we then produce a clustering partition of the sessions to identify and characterize different types of browsing activity. This will lead us to quantify the relation between types of browsing and diversifying patterns. Then, we discuss these results and suggest possible explanations for the patterns observed, and finally conclude on the applications of these analyses to recommender systems and website design.

\section{Related Work}

The diversity of information consumption on the web (e.g. press articles, commercial items, posts on social media) has attracted growing attention in recent years. Different diversity measures have emerged as useful tools to describe pressing issues related to phenomena such as filter bubbles, echo chambers, and the development of extreme opinions [Par11]. In commercial applications, such as designing and improving recommender systems, diversity is the focus of increasing interests as it relates to user satisfaction, exposure to new relevant products, and even customization and context-aware platforms $\left[\mathrm{A}^{+} 16\right.$, Section 7.3]. Several previous works study the characterization of the diversity of information consumption on social platforms such as Twitter $\left[\mathrm{BJN}^{+} 15\right]$, Facebook $\left[\mathrm{SZDV}^{+} 17\right]$, and from news outlets $\left[\mathrm{KZN}^{+} 15\right.$, FGR16]. In commercial applications, diversity has come to be seen as an integral part of users' satisfaction [RRS15, ZKL $\left.{ }^{+} 10, \mathrm{FH} 09, \mathrm{VC} 11\right]$ and its measurement has become a tool in the search of a broader understanding of browsing behaviors, e.g. automatic detection of change of context [LCB16].

The study of web logs is a field of research that counts a wealth of works [Jan08, Pet93, ACDN12, SMHM99], and we cannot aim at providing a survey on the topic in this article. The use of web log analyses to gain insight on 
how the structure of a website affects user navigation is a well established domain of research. Several studies investigate how the structure of a website influences the ability of users to obtain desired resources [HSGS13, McA89, NHW05, MS98]. Other studies have focused on the relation between the structure of websites and browsing paths taken by users $\left[\mathrm{NDBB}^{+} 19\right.$, DSLS17, Wol08, SPWL14, PWZL16, WL12].

Systematic web log analysis has allowed for improvements in the design of websites [CPP00], predicting the resource a user is looking for or the location in which it is expected to be found [SY01, TASJ14]. Web log analysis also offers the possibility of dynamic and/or personalized adjustments to the structure of websites [CR13, FSCJ02]. While most advancements make use of web logs, or even of contents of pages in a site [LK07], fewer studies use the logs to address the question of the diversity of the contents browsed. This question has been recently related to filter bubbles [ $\left.\mathrm{NHH}^{+} 14, \mathrm{NOFM} 15\right]$. Some studies focus on navigation volume [MF01] and on the diversity of types of users and navigation patterns [NHJ08], but not so many on the diversity of consumption itself [OCJ12].

Studies addressing the question of consumed content diversity in browsing and site structure should account for ways of classifying pages according to content and to their role in the site structure. Some classifications of pages in web logs include information such as differentiation of social platforms or search engine pages [TASJ14]. Our work tackles specifically the question of the influence of the website structure on the diversity of information consumption by users. In this study, diversity refers to a measure of variety and balance (in this case Shannon Entropy) of the topics consumed by a user while browsing a website in which each page can be classified as dealing with a given topic. In contrast with much of previous works, here the structure will be considered to be a given and not something to be learned from web log analysis: pages in the website have a fixed role within the structure (e.g. menu pages, articles, ....).

\section{Dataset and diversity computation}

\subsection{Data general description}

The data used for the study of browsing properties within a website is most often available in the form of a log file created by a web server which contains 
information about the requests made. Commonly used formats provide for each request, among other information: IP address of the client, the method and the resource requested from the client, the HTTP status code returned, the size of the object returned, and the timestamp of the request.

For our case study, we use the log files of a particular website: Melty (www . melty.fr), a French information and entertainment website targeting the 1534 year-old demographics. From the data available to us, we chose a subset corresponding to 3 weeks of September 2017 as it was deemed sufficiently extensive for the illustration of the present analyses ${ }^{1}$. Tests made with other time periods yield similar results to what is presented in the following.

A general description of the structure of www.melty.fr is useful for the rest of our study. An examination of the site reveals that it is organized in a tree-like fashion. From the home page, users can access thematic menu pages (topic pages) for broad topics (such as music or TV), as well as more specific subtopic pages, or articles. Note also that links to menu pages are always present in the menu bar of most pages, which allows in theory for users to go to these menu pages from nearly anywhere in the site. From the topic pages, access is offered to other, more specific menu pages (subtopic pages) dedicated to the same topic, but with more specific content (e.g., a given band for the music topic). And from these specific pages, a user has access to various pages with different types of roles (e.g. articles, media pages, forums, photo galleries). This classification in terms of structural role is predefined in the website design.

Let us now describe a typical article page on the website. In addition to the links accessible via the menu bar, a user may navigate through the website using various kinds of links. First, some links are anchored in the text of the article itself and are therefore static. The bottom part of an article is dedicated to dynamic recommendation links, which either connect to external websites, or to other Melty pages.

\subsection{Preprocessing and sessionization}

Throughout our study we identify a user with a hash, combining their IP, logname, username, and session cookie. In the data available to us, a hash function for creating user IDs in the log had already been applied by Melty, rendering users anonymous so as to answer privacy concerns. The web server

\footnotetext{
${ }^{1}$ The anonymized dataset is available at http://data.complexnetworks.fr/melty/
} 
also complements the log information with the referral page of each request. This is useful for two reasons: it provides information about the paths followed by users along a session (for instance when they open browser tabs or windows by following several links from a same page), and it also gives information about where the users come from, in particular from a search engine or from a social media platform.

In order to analyze the log file, we must first discard the requests originating from non-human agents. For this purpose, we take a standard mixed approach, using syntactical log analysis and traffic pattern analysis [DG11]. Firstly, in a syntactical log analysis, we delete requests made by user agents known to be used by common robots. Secondly, in a traffic pattern analysis, we filter out requests from the log using some activity-based criteria taking elements from [TK04, BGST05]. Concretely, we excluded requests by users that requested more than 20 pages per minute or more than 900 pages per hour, as well as requests by users that were active for 20 or more consecutive hours.

We analyze the log at the session level rather than at the user level, our motivation being that a user may display different browsing behaviors in different sessions. For this purpose, we sessionize the log data. While there is no clear consensus about how to do so [WC16, JK08], a reasonable strategy $\left[\mathrm{HKK}^{+} 15\right]$ makes use of a cutoff sessionization time. This time interval cutoff has been often found to be around 30 minutes, which is often used as a standard sessionization time parameter.

\subsection{Data representation}

We define here some general notations which are used in this work. Given a $\log$ file, we identify the set $V$ of web pages and the set $\mathcal{S}$ of sessions present in it. $T$ denotes the set of timestamps at which the requests were made. The comprehensive set $\mathcal{R}$ of requests in the log is such that $\mathcal{R} \subseteq V \times V \times \mathcal{S} \times T$. For every request $\ell$ in a $\log \mathcal{R}$ we consider its source $v_{s}(\ell) \in V$, i.e. the page where the request originated, its target $v_{t}(\ell) \in V$, i.e. the requested page, and time $t(\ell) \in T$ of the request. Table 1 and Figure 1 show a descriptive summary of the main parameters of the logs, after preprocessing and sessionization.

In the following, we use the concept of session graphs. A session graph is a directed graph, which nodes are pages browsed during a session, and a directed link corresponds to the request $\ell$ made by the user from a source page $v_{s}(\ell)$ to a target page $v_{t}(\ell)$. Describing a session with a graph - rather 
Table 1: Descriptive summary of the sessionized $\log \mathcal{R}$.

\begin{tabular}{|c|c|c|}
\hline Log start & $\min \{t(\ell): \ell \in \mathcal{R}\}$ & $2017-09-0400: 12: 05$ \\
Log end & $\max \{t(\ell): \ell \in \mathcal{R}\}$ & $2017-09-2423: 59: 58$ \\
\# Requests & $|\mathcal{R}|$ & $14,977,605$ \\
\# Sessions & $|\mathcal{S}|$ & $8,470,403$ \\
\# Pages & $|V|$ & 269,257 \\
\hline
\end{tabular}

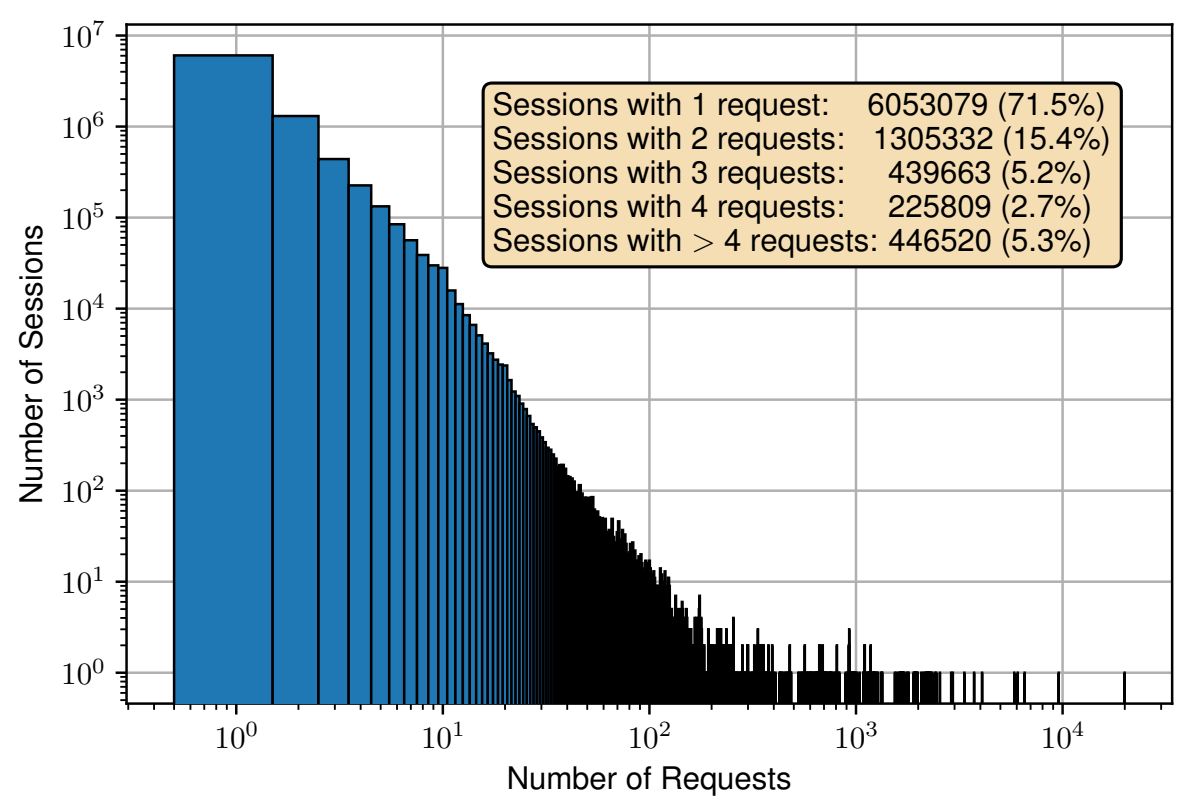

Figure 1: Distribution of the number of sessions for a given number of requests in the dataset.

than a sequence - allows to distinguish different types of browsing behaviors, as we shall see in the following.

\subsection{Twofold page classification}

It is often possible to describe the pages of a website according to a classification which has two dimensions: one describing the structural function of a page, and the other its content. In some cases, typically commercial 
websites, the content classification (e.g. furniture, electronics, books, films) is tightly related to the structure. Products are organized in categories according to a tree-like structure, so that the website favors browsing within a same category. In other cases, e.g. Wikipedia, the relation is much more loose: a user has a high probability to change content category (e.g. mathematics, history, biology) by following a hyperlink. Depending on the case under examination, the relation between the structural role of a page and its content may differ. In particular the possibility to navigate from a topic to another largely depends on design choices, as we will discuss in this study.

Our dataset exhibits such a classification, pages have an associated content type (classifying the topic with which they deal, such as TV or Video games), and a functional category type (classifying the role that they play in the site, such as articles or forum pages). We naturally consider the diversity of page consumption in regards to their content. We denote the set of topics available by $A$, and the corresponding classification function $C_{A}: V \rightarrow A$, which assigns to each element of $V$ a topic in $A$. Similarly, pages are assigned a structural role, which is an element of the role set $B$, corresponding to the classification function $C_{B}: V \rightarrow B$.

In the Melty website that is our case study, we have:

- content type set $A=\{$ Celebrities, Comics, Movies, Music, News, Series, TV, VideoGames, Other $\}$,

- structural role set $B=\{$ Article, Forum, Gallery, Quiz, Subtopic page, Topic page, Other\}.

Pages which are $B$-labeled Topic page are menu pages for general topics in $A$, while pages which are $B$-labeled Subtopic pages are menu pages for more specific subjects within a topic in $A$. For example, the topic Music may have its own menu page of $B$-labeled Topic page, while some more specific subjects such as rock music could have their own menu page with B-label Subtopic page.

\subsection{Consumption diversity computation}

Whenever we have a subset of a $\log , \mathcal{L} \subseteq \mathcal{R}$ (for example, the log of a user, or the log of a session), we can compute its diversity with respect to a page classification, e.g. $C_{A}$, using Shannon entropy: 


$$
\begin{gathered}
\mathcal{D}_{A}(\mathcal{L})=-\sum_{a \in A} p_{A}(\mathcal{L}, a) \log _{2} p_{A}(\mathcal{L}, a), \\
\text { with } p_{A}(\mathcal{L}, a)=\frac{\left|\left\{\ell \in \mathcal{L}: C_{A}\left(v_{t}(\ell)\right)=a\right\}\right|}{|\mathcal{L}|} .
\end{gathered}
$$

Given the Shannon entropy $E$ of the information consumption related to some subset of requests $\mathcal{L}$, we define its Iso-Entropic Uniform Consumption (IEUC) diversity index as $2^{E}$. This quantity is also known as perplexity in information theory [BJM83]. The interest of quantifying diversity with IEUC is that it can be interpreted as the number of consumed types needed to produce the same entropy if the consumption was uniform, meaning that for any two types $a, a^{\prime}$ in $A$, we would have $p_{A}(\mathcal{L}, a)=p_{A}\left(\mathcal{L}, a^{\prime}\right)$.

While other diversity metrics exist (Richness, Herfindhal Index, and Gini Index are notable examples), Shannon entropy (or simply the entropy) is a popular choice [NOFM15, EMC10, PSL13] because it accounts for the variety of types of elements (i.e. $|A|$, the size of $A$ ) and for the balance among them (i.e. the comparison between different values $p_{A}(\mathcal{L}, a)$ for different $a \in A$ ) while proving meaningful interpretations, see [Sti98, Chapter 2] for a more detailed discussion. In the following, we refer to the entropy measurement as consumption diversity, as it reflects the variety of topics that a user goes through during a session on Melty website.

\section{Aggregated consumption diversity}

In order to get insights about some general features of the log dataset regarding consumption diversity, we first obtain some measurements on subsets of the log file, grouped according to the number of requests in a session.

As expected when measuring aggregated human behavior on a website, we can see in Figure 1 that the number of requests per session is distributed heterogeneously, roughly following a power-law. Consequently, a majority of sessions $(6,053,079$, that is $71.5 \%$ of them) consist of only one request (i.e. requesting a single page without further navigating within the website). Then, the number of sessions with a given number of requests decreases, with only $5.3 \%(446,520)$ requesting more than 4 pages. Figure 2 shows how the content types vary according to the number of requests. It also specifies the associated entropy and IEUC. It can be observed that diversity decreases 
when the number of requests per session increases, therefore indicating that longer sessions tend to focus on the same type of content (and above all, TV).

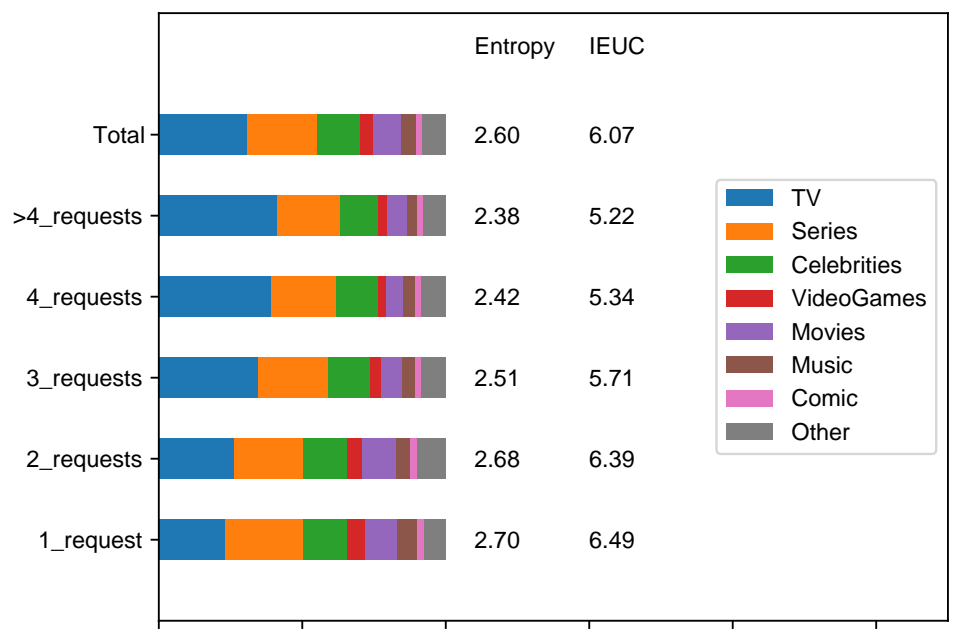

Figure 2: Topic consumption and diversity in sessions aggregated by number of requests.

Concerning the evolution of consumption diversity through time, the log presents regularities and particularities that we discuss here. For illustrative purposes, let us consider the week spanning from $9 / 18 / 2017$ to $9 / 24 / 2017$, and let us investigate the consumption volume and diversity of different groups of sessions, as shown in Figure 3.

When grouping sessions by number of requests, we observe that activity measures present some regularities in the hourly volume of requests, displaying growing activity during most of the day until spiking later before midnight. The evolution of the hourly diversity for the complete log is comparable to that of the group of sessions with more than 4 requests, although the value of the diversity of this higher engagement group is generally lower, in accordance with what was observed in Figure 2.

We also separate sessions by origin, distinguishing sessions which originate either from a search engine or from a social platform. Strong differences can be observed. Indeed, the consumption diversity of sessions originating from social platforms is noticeably lower than that of other groups. This type of phenomenon had been observed previously in [NOFM15]. Note that 


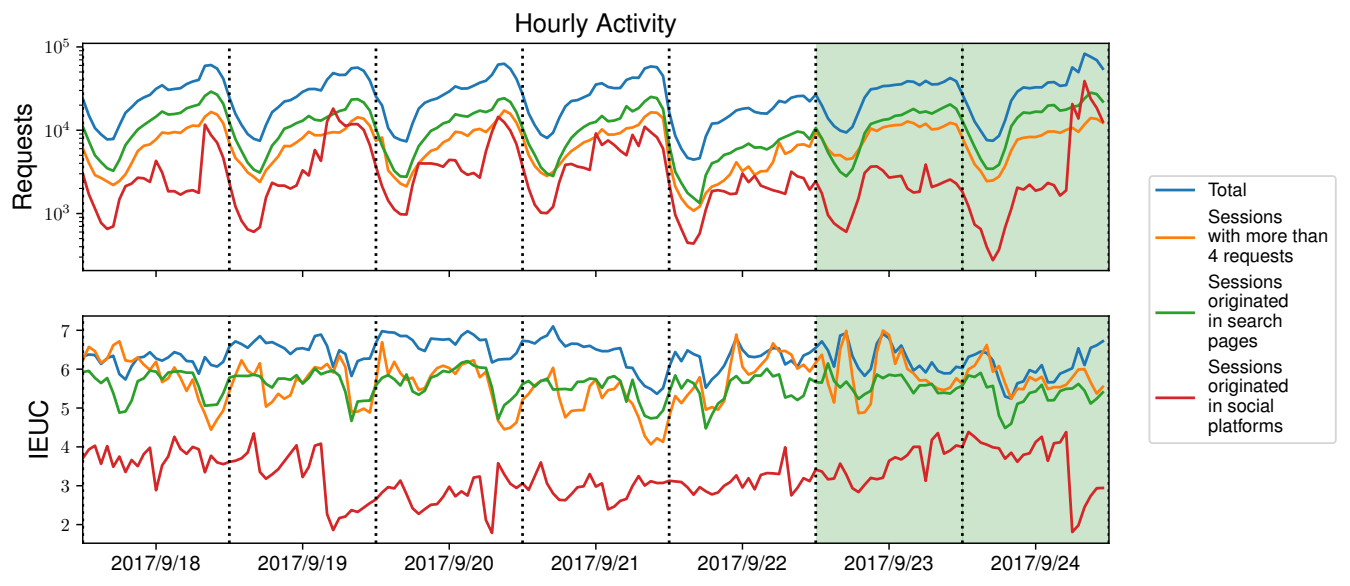

Figure 3: Hourly activity (top) and diversity (bottom) over time of the log divided into groups of sessions, depending on the number of requests, or on the origin of the sessions (social or search pages).

diversity as measured by entropy or IEUC is not affected by the volume of consumption, meaning that the fact that there are less sessions originating from social platforms should not affect the diversity measurements. The sudden diversity losses for browsing originating in social platforms are the consequence of surges of activity around specific content types. Figure 4 illustrates this effect through a more detailed examination of one of these events: diversity loss observed on the afternoon of September 19th 2017 for consumption by sessions originated in social platforms is related to a surge of activity focusing on the content type Movies.

Figure 5 further explains the diversity loss event. We measure the activity of the five most popular pages in browsing originated in social platforms around the time of the event. It reveals that a Melty page about a movie was abruptly requested in sessions coming from social platforms. In this particular case, the page is about Harry Potter movies, and traffic came from Facebook.

Finally, let us consider the weighted directed graph constructed by aggregating the whole log, sometimes referred to as browsing graph [TASJ14]. Its nodes are pages, directed edges represent requests from a source to a target page, and their weight is the number of times an edge was taken. Over this aggregated graph, we compute topological indices reflecting nodes centrality for each page: in- and out-degree, as well as betweenness centrality, calcu- 

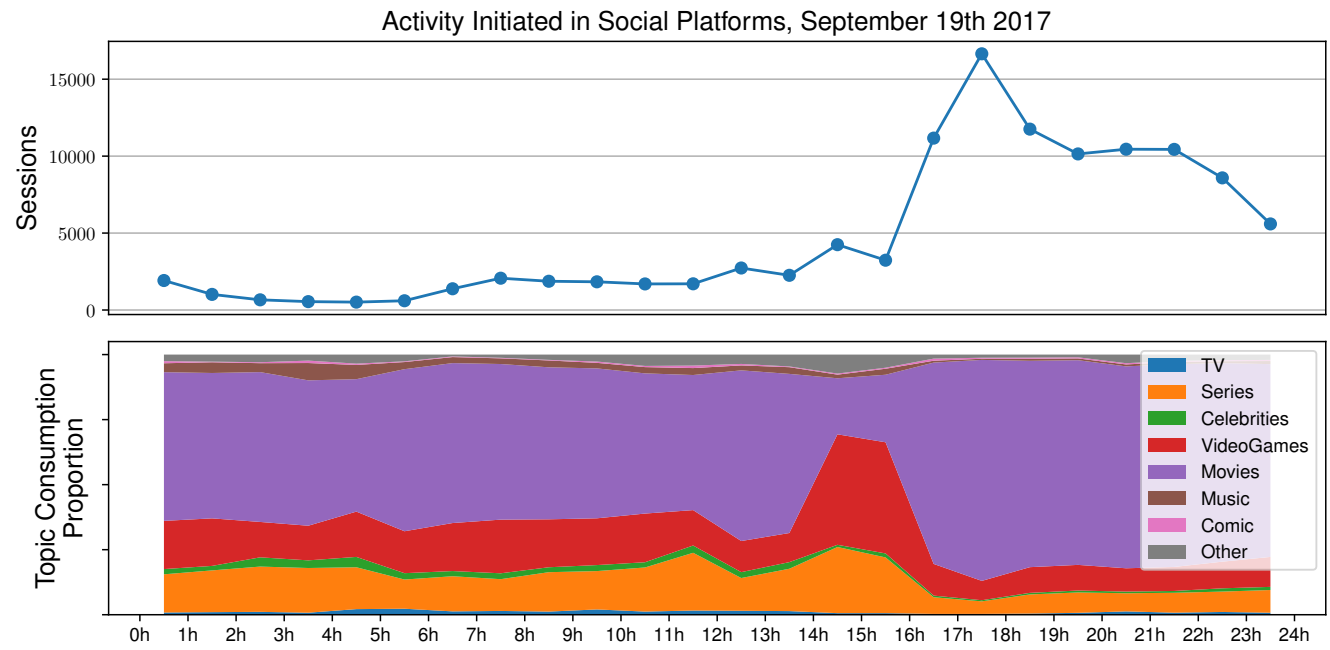

Figure 4: Hourly activity of navigation originating in social platforms on September 19th, 2017. Number of sessions with origin in social platforms present (top), and topic consumption proportion $p_{A}$ for content types in $A$ (bottom).



Figure 5: Number of requests per minute, around the time of the diversity loss event of September 19th, 2017, for the five most popular pages (and their content type) in the browsing activity of sessions originated in social platforms.

lated using [Bra01]. We then group pages by structural role (classification $B$ ) and represent their Complementary Cumulative Distribution Function (CCDF) in Figure 6. We observe that topic pages clearly have a higher probability of having a high in- and out-degree, or betweenness centrality value than subtopic or article pages. This suggests that pages with different 
structural role labels seem to have different functions during navigation, as expected. Indeed, some pages tend to be navigation end pages (e.g. articles) while other act more like transit hubs (topic pages).
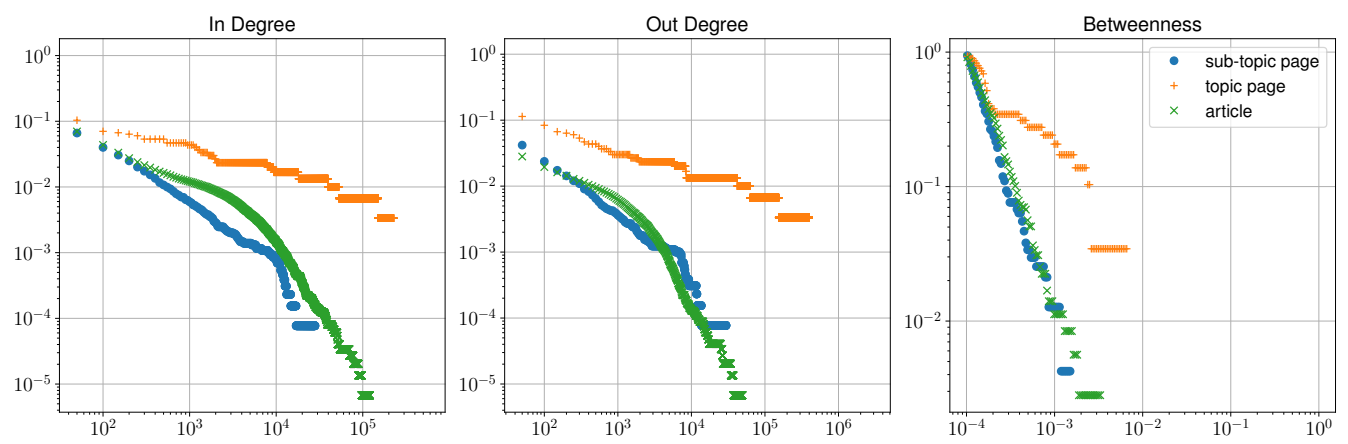

Figure 6: Complementary Cumulative Distribution Functions (CCDF) of pages centrality index, depending on their structural role.

These elements motivate the description of the variety of sessions and the analysis of the diversification patterns associated with types of pages and groups of sessions.

\section{Consumption diversity in session clusters}

In this section, we define clusters which correspond to distinct subgroups of browsing behaviors in the data. Precisely, we investigate how the consumption diversity of sessions is related to different features that characterize the browsing behavior of users, such as click frequency and tendency for multitabbing. For this purpose, we focus on sessions that contain more than 4 requests, as they bring sufficient information per session, without filtering too many sessions out of the data log. Indeed, we want to include structural information related to the session graphs in the clustering process, which demands to have several requests per session.

We aim at separating sessions into different groups according to quantitative properties. The features that we have selected for this purpose are known from previous works to be relevant features for session clustering [Ste08, CC01], namely:

- number of requests, 
- duration of the session,

- mean time between requests,

- standard deviation of the time between requests.

We also include a feature that describes the structure of the directed graph which represents the session. We use the star-chain index, adapted from [BTLG13], which is computed on the directed graph $\mathcal{G}=(V, E)$ of each session as:

$$
\text { star-chain index }=\frac{\sum_{v \in V, d_{o}(v)=0} d_{i}(v)-1}{\sum_{v \in V} d_{i}(v)-1},
$$

where $d_{i}(v)$ and $d_{o}(v)$ are the in- and the out-degrees of node $v$. The starchain index is closer to 0 whenever the session graph has a chain-like form, and closer to 1 whenever a session graph rather resembles a star: one single node serving as the source of access to all others. Higher values of the starchain index signal either a higher level of multi-tabbing in browsing, or a frequent use of the back button to revisit specific pages.

In the resulting feature space, we apply standard clustering techniques. We first perform a normalization on the various dimensions of the feature space. Precisely, all selected features exhibit a long-tailed distribution except the star-chain index, thus we apply a logarithmic transformation in order to avoid the outliers to play an overwhelming role in the clustering. Then, we normalize the values to obtain data with unit variance on each feature. We use a weighted k-means [GGTN17] clustering by stages, following [CC01], to cluster first in the space of the dimensions which are not related to the topology of the session graph (i.e. duration, number of requests, mean and standard deviation of time between requests) and then to cluster in the dimension of the star-chain index. We present the results for a clustering in 6 clusters or groups. Different choices for the number of groups tend to show similar trends, so it has been chosen as the lowest value which allows to clearly separate qualitatively distinct browsing behaviors in relatively balanced clusters, as we shall see.

In order to summarize the results of the clustering, we show in Figure 7 the centroids of each cluster in the plane spanned by the first two principal components axes (PC-1 and PC-2) that better explain the variance of session features in the feature space, as computed using Principal Component 
Analysis (PCA). We observe that the first dimension is clearly defined by the star-chain index, while the second is dominated by session duration and number of requests.
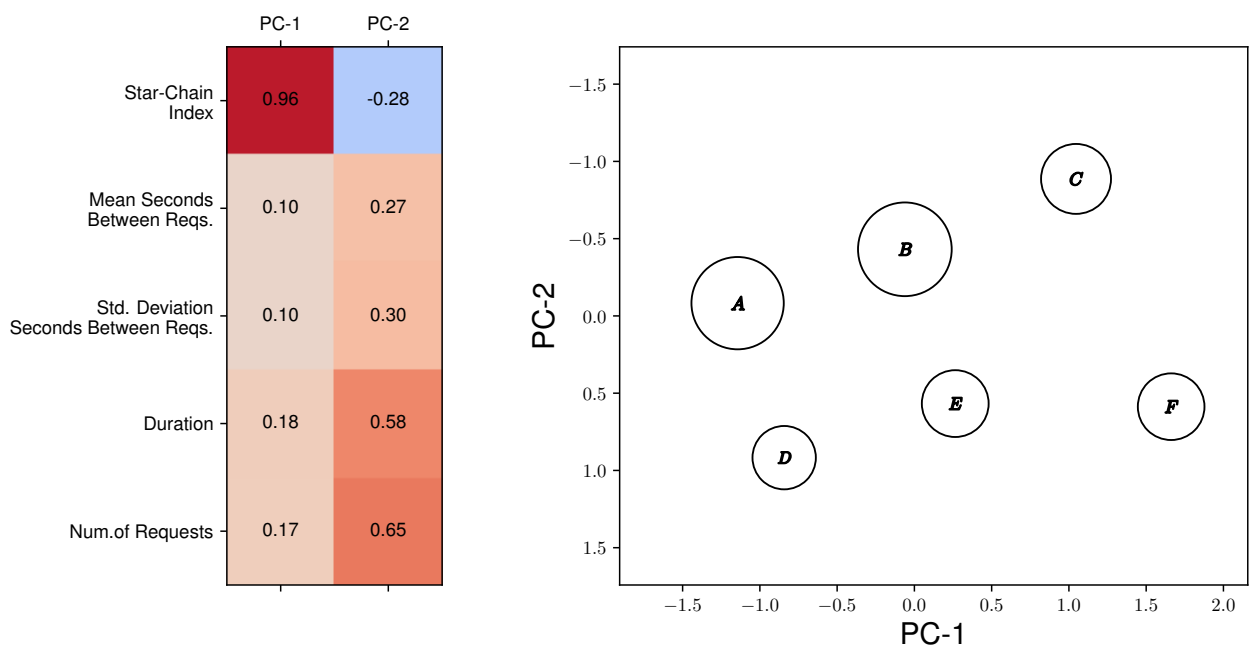

Figure 7: Composition of the first two Principal Components of the PCSpace of the features space (left), and the positions of the cluster centroids (right), with areas proportional to the number of sessions inside each cluster.

Figure 8 shows a random sampling of 5 sessions per cluster, illustrating qualitatively how these dominating features define each cluster. A more quantitative depiction of the characteristics of each cluster in the feature space is provided in Figure 9, which shows the distributions of the features for each cluster using box plots.

More importantly, Table 2 summarizes relevant statistics for each cluster, in particular their sizes and consumption diversities. It can be seen that, among sessions with more than 4 requests, higher diversities tend to be related to more star-like sessions and to a lesser extent, longer sessions with more requests (which is not surprising). This suggests that star-like browsing is related to the possibility of switching topics more easily on this website. Another interesting observation is that sessions originating from a social network tend to be more star-like than chain-like. On the other hand, sessions originating from search engines tend to be more chain-like than star-like. However, we have previously observed that the later kind of sessions had higher diversity, as a group, than the former. These observations indicate 

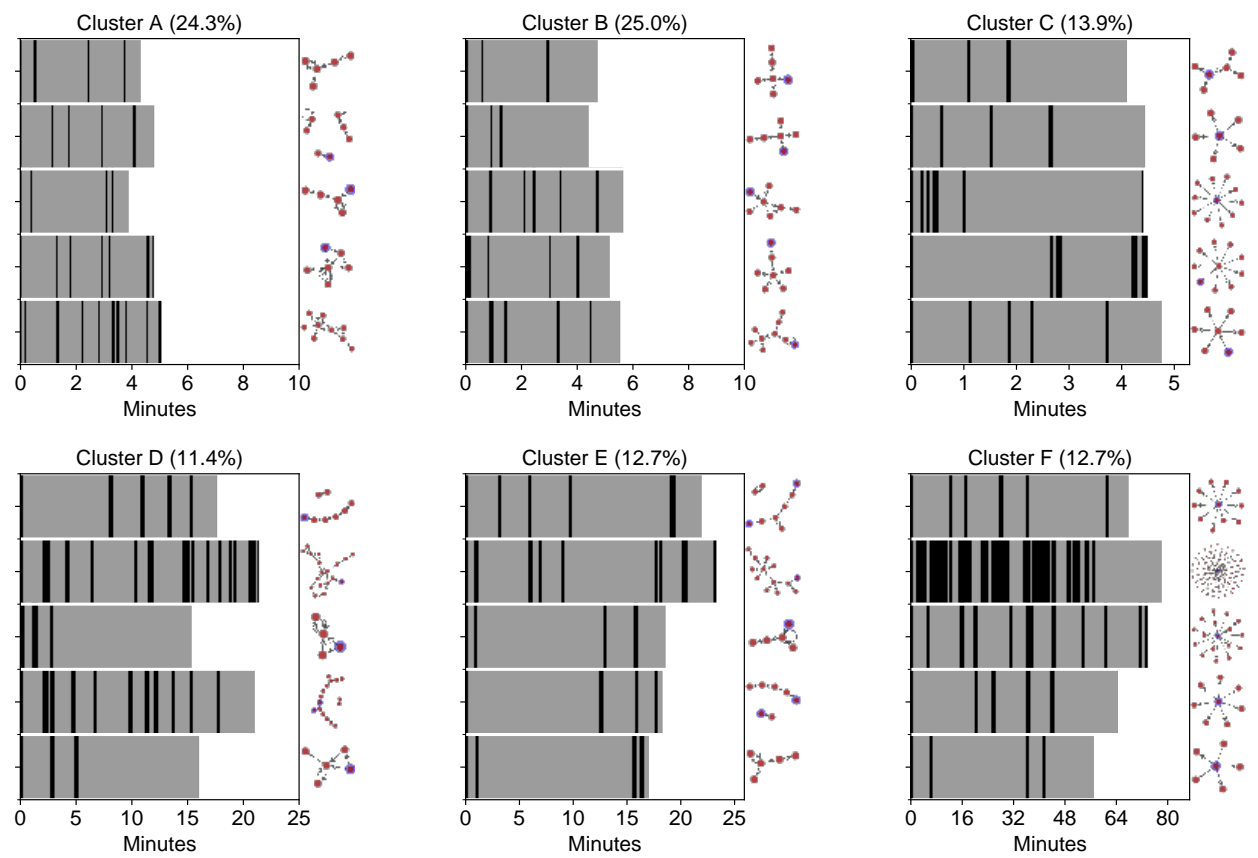

Figure 8: Random samples of sessions in each cluster. Each session is portrayed as a time line, with black lines marking the instants at which requests are made, next to the graph representing the session.
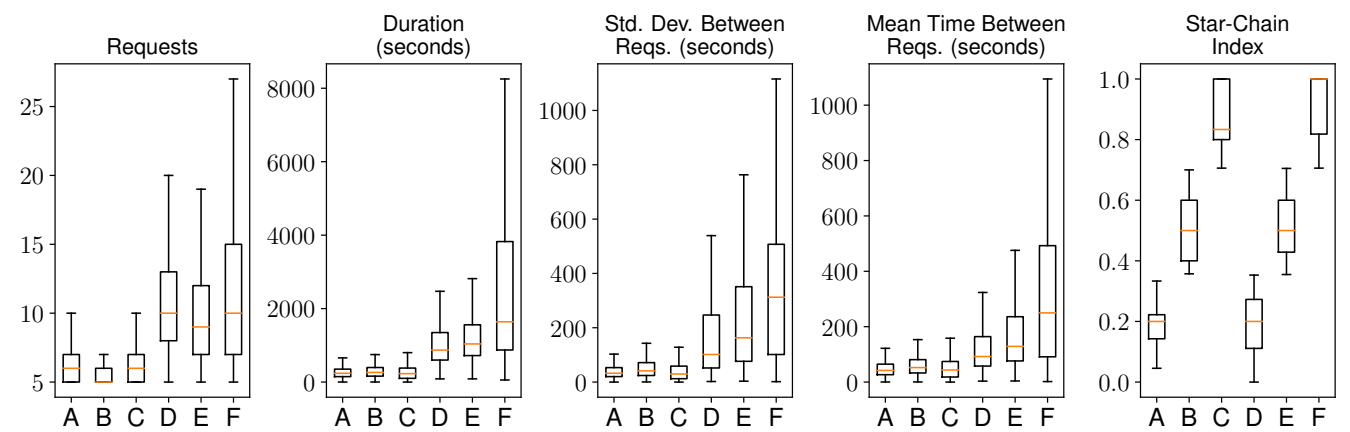

Figure 9: Box plots showing the distribution of the selected sessions features in each cluster.

not only that users behave differently depending on how they arrived on the website, but also that among each of the subgroups, a refined typology of browsing behaviors would be certainly useful. 
Table 2: Sizes and diversities (entropy and IEUC) of the identified clusters, with the percentages of of many of the sessions of the cluster were originated in search engines and in social platforms.

\begin{tabular}{|c|c|c|}
\hline $\begin{array}{l}\text { Cluster A } \\
108,391 \text { Sessions }(24.3 \%) \\
\text { Entropy }=2.2 \\
\text { IEUC }=4.6 \\
\text { Search Sessions }=42.6 \% \\
\text { Social Sessions }=1.1 \%\end{array}$ & $\begin{array}{l}\text { Cluster B } \\
111,624 \text { Sessions }(25.0 \%) \\
\text { Entropy }=2.3 \\
\text { IEUC }=4.9 \\
\text { Search Sessions }=48.7 \% \\
\text { Social Sessions }=1.2 \%\end{array}$ & $\begin{array}{l}\text { Cluster C } \\
62,204 \text { Sessions }(13.9 \%) \\
\text { Entropy }=2.3 \\
\text { IEUC }=4.9 \\
\text { Search Sessions }=36.3 \% \\
\text { Social Sessions }=7.6 \%\end{array}$ \\
\hline $\begin{array}{l}\text { Cluster D } \\
51,059 \text { Sessions }(11.4 \%) \\
\text { Entropy }=2.1 \\
\text { IEUC }=4.3 \\
\text { Search Sessions }=51.1 \% \\
\text { Social Sessions }=1.3 \%\end{array}$ & $\begin{array}{l}\text { Cluster E } \\
56,762 \text { Sessions }(12.7 \%) \\
\text { Entropy }=2.4 \\
\text { IEUC }=5.3 \\
\text { Search Sessions }=55.3 \% \\
\text { Social Sessions }=2.0 \%\end{array}$ & $\begin{array}{l}\text { Cluster } \mathbf{F} \\
56,480 \text { Sessions }(12.6 \%) \\
\text { Entropy }=2.7 \\
\text { IEUC }=6.5 \\
\text { Search Sessions }=27.8 \% \\
\text { Social Sessions }=6.2 \%\end{array}$ \\
\hline
\end{tabular}

To get a clearer picture of the impact of the browsing behaviors on consumption diversity, we now turn our attention to the patterns that are related to diversification within each group of the partition achieved through clustering.

\section{Diversifying requests}

The diversity of a session is related to the variety of contents of the pages requested during that session. This is why we take interest in navigation patterns that produce changes in content types according to the contentbased classification. More specifically, we are interested in measuring how the structural role of a page (its label according to classification $C_{B}$ ), relates to changes in the topic (its label according to classification $C_{A}$ ). We obviously limit this analysis to the requests that link two pages inside Melty (source pages from outside - such as search engines or social platforms - are excluded). Also, we only consider the requests made during sessions with more than 4 requests, once again. Therefore the following analysis is performed over a subset of the original log, containing 2,064,552 requests and 380,506 sessions.

Given this subset $\mathcal{L}$ of the original log, let us consider the subset of requests going from a page with type (i.e., functional role) $i \in B$ to a page with type $j \in B$, as follows:

$$
\mathcal{L}_{i \rightarrow j}=\left\{\ell \in \mathcal{L}: C_{B}\left(v_{s}(\ell)\right)=i \wedge C_{B}\left(v_{t}(\ell)\right)=j\right\}
$$


Thus, we estimate the probability $P_{i \rightarrow j}$ that a given link goes from a role type $i$ to a role type $j$ in $B$ as $P_{i \rightarrow j}=\left|\mathcal{L}_{i \rightarrow j}\right| /|\mathcal{L}|$. We call the associated probability matrix the Browsing Pattern matrix. Note that this is different from the probability associated to the empirical Markovian matrix for $\mathcal{L}$, which gives the probability, knowing the source node type $i$, to transit to a target node with type $j$.

Next, for a request $\ell$ going from a page of type $i \in B$ to a page with type $j \in B$, we are interested in estimating the probability that it produces a change of content type in $A$. Therefore, we estimate, for all $i, j \in B$, the probabilities:

$$
P_{i \rightarrow j}^{\operatorname{trans}(A)}=\mathbb{P}\left[i \rightarrow j, C_{A}\left(v_{s}(\ell)\right) \neq C_{A}\left(v_{t}(\ell)\right)\right] .
$$

Using the subset $\mathcal{L}$ of the $\log$ as an empirical observation we estimate these probabilities as

$$
P_{i \rightarrow j}^{\text {trans }(A)}=\frac{\left|\left\{\ell \in \mathcal{L}_{i \rightarrow j}: C_{A}\left(v_{s}(\ell)\right) \neq C_{A}\left(v_{t}(\ell)\right)\right\}\right|}{\left|\mathcal{L}_{i \rightarrow j}\right|} .
$$

We call the associated probability matrix the Diversifying Pattern matrix. We show the values computed on the log in Figure 10, arranged as matrices taking the role classification set $B$ as indices. Some transitions between $B$ type categories are very rare. Thus, we set a threshold of 50 observations (requests) in the estimation of probability $P_{i \rightarrow j}^{\operatorname{trans}(A)}$. When a transition does not reach this threshold, the estimation was not considered (marked with an $\mathrm{X}$ in the matrix) to avoid misinterpretations.

Let us first underline some characteristics of the global Browsing Pattern matrix over the data log shown in the upper part of Figure 10. Most requests go from an article to another article (that is $57.1 \%$ of the traffic), followed by requests from topic pages to articles (18.7\%), and then by requests from subtopic pages to articles $(6.7 \%)$. Now the Diversifying Pattern matrix (bottom part of the same figure) shows that the requests that most changed in content type go from topic pages to other topic pages $(67.6 \%$ of the times, a topic transition occurred). More generally, requests to topic pages and, to a lesser extent, to subtopic pages, are often involved in topic transitions. Also, the Browsing Pattern matrix indicates that requests from topic and subtopic pages represent an important fraction of the traffic on the website. Topic pages in particular tend to have the role of hubs, as observed in Sec. 4. Thus, we can draw a first basic picture from these observations: users usually 


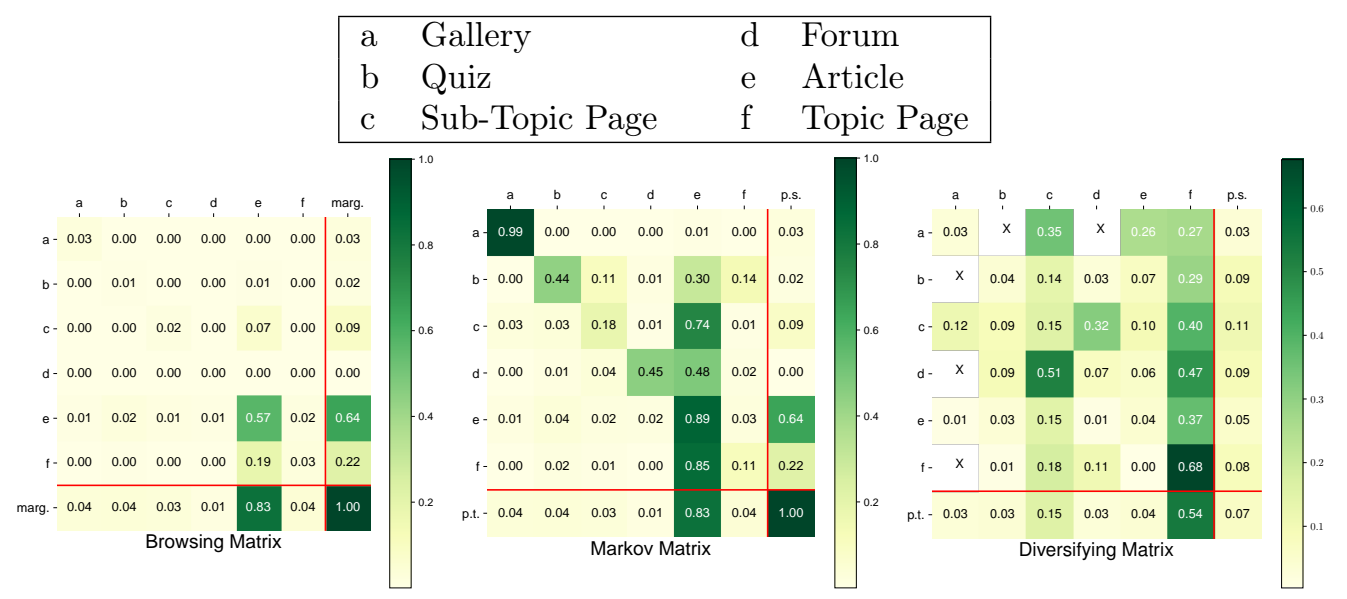

Figure 10: Browsing Pattern matrix, associated with $P_{i \rightarrow j}$ (left), its associated Markovian matrix (middle), and Diversifying Pattern matrix associated with $P_{i \rightarrow j}^{\operatorname{trans}(A)}$ (right). The marginals of each line and column are given respectively to the right and below each matrix. Probability of content transition per type of source, and per type of target pages are indicated respectively as p.s and p.t.

navigate from article to article but sometimes change topics by moving to topic and subtopic pages, from where they find new articles.

While this sketches a general picture of the traffic of users across the site structure and the mechanisms through which diversification occurs, we are further interested in differentiating these mechanisms for groups of sessions. The same measures, listing the first 3 browsing and diversifying kinds of requests per cluster yields the results shown in Table 3.

The most salient observation that we can make from Table 3 is that the dominating types of requests differ from a cluster to another one. More precisely, while clusters featuring mostly chain-like sessions tend to be dominated by article to article requests, clusters featuring mostly star-like sessions tend to be dominated with transitions from topic pages to articles. Concerning the diversification patterns, the observations per cluster are consistent with the general trend, that is to say that topic (or subtopic) pages are involved either as sources, or as targets, or even both in all major diversifying requests.

We now examine the Browsing Pattern and the Diversifying Pattern matrices of the activity of the session with more than 4 requests, divided into 
Table 3: Clusters identified by dominating characteristics showing the main browsing and diversification patterns.

\begin{tabular}{|c|c|c|c|c|c|}
\hline Cluster & IEUC & star or chain & length & $\frac{1}{\text { Browsing Pattern }}$ & Diversification Pattern \\
\hline A & 4.6 & chain & short & $\begin{array}{l}75 \%: \text { article } \rightarrow \text { article } \\
5 \%: \text { topic page } \rightarrow \text { article } \\
4 \%: \text { gallery } \rightarrow \text { gallery }\end{array}$ & $\begin{array}{l}70 \%: \text { topic page } \rightarrow \text { topic page } \\
42 \%: \text { subtopic page } \rightarrow \text { topic page } \\
36 \%: \text { article } \rightarrow \text { topic page }\end{array}$ \\
\hline B & 4.9 & mixed & short & $\begin{array}{l}51 \%: \text { article } \rightarrow \text { article } \\
23 \%: \text { topic page } \rightarrow \text { article } \\
8 \%: \text { subtopic page } \rightarrow \text { article }\end{array}$ & $\begin{array}{l}71 \%: \text { topic page } \rightarrow \text { topic page } \\
44 \%: \text { article } \rightarrow \text { topic page } \\
39 \%: \text { subtopic page } \rightarrow \text { topic page }\end{array}$ \\
\hline C & 4.9 & star & short & $\begin{array}{l}63 \%: \text { topic page } \rightarrow \text { article } \\
21 \%: \text { article } \rightarrow \text { article } \\
10 \%: \text { subtopic page } \rightarrow \text { article }\end{array}$ & $\begin{array}{l}65 \%: \text { topic page } \rightarrow \text { topic page } \\
39 \%: \text { article } \rightarrow \text { topic page } \\
28 \% \text { : subtopic page } \rightarrow \text { topic page }\end{array}$ \\
\hline D & 4.3 & chain & medium & $\begin{array}{l}71 \%: \text { article } \rightarrow \text { article } \\
7 \%: \text { topic page } \rightarrow \text { article } \\
5 \%: \text { gallery } \rightarrow \text { gallery }\end{array}$ & $\begin{array}{l}62 \%: \text { forum } \rightarrow \text { sub-topic page } \\
62 \%: \text { topic page } \rightarrow \text { topic page } \\
46 \%: \text { forum } \rightarrow \text { topic page }\end{array}$ \\
\hline $\mathrm{E}$ & 5.3 & mixed & medium & $\begin{array}{l}47 \%: \text { article } \rightarrow \text { article } \\
23 \%: \text { topic page } \rightarrow \text { article } \\
11 \%: \text { subtopic page } \rightarrow \text { article }\end{array}$ & $\begin{array}{l}65 \%: \text { topic page } \rightarrow \text { topic page } \\
46 \%: \text { article } \rightarrow \text { topic page } \\
46 \%: \text { forum } \rightarrow \text { sub-topic page }\end{array}$ \\
\hline $\mathrm{F}$ & 6.5 & star & long & $\begin{array}{l}43 \%: \text { topic page } \rightarrow \text { article } \\
24 \%: \text { article } \rightarrow \text { article } \\
17 \%: \text { subtopic page } \rightarrow \text { article }\end{array}$ & $\begin{array}{l}69 \%: \text { topic page } \rightarrow \text { topic page } \\
48 \%: \text { article } \rightarrow \text { topic page } \\
43 \% \text { : subtopic page } \rightarrow \text { topic page }\end{array}$ \\
\hline
\end{tabular}

three groups: 1) Native: sessions that start navigation directly within the website, 2) Search: sessions that start navigation from a search engine, and 3) Social: sessions that start navigation from a social platform. Table 4 shows the summarized characteristics of these 3 groups. While the Browsing Patterns of the Native and Search groups are relatively similar, the Social group stands out. As mentioned before, sessions coming from social platforms have a lower consumption diversity, as they tend to make less use of the website menus to explore different topics. Indeed, these sessions do not have a lot of requests using topic or subtopic pages, but when they do, they have a high probability of changing topic. This can be observed in the Diversifying Pattern count, also portrayed visually in Figure11. A hypothetical interpretation is that users coming from a social platform comes to Melty to see a specific content. Most of the times they leave the platform after checking it, but sometimes they discover an unexpected link to another topic, which also raises their interest. If this is true, it could be described as a serendipitous discovery.

Table 4: Group decomposition of sessions with more than 4 requests according to origin of navigation showing browsing and diversification patterns.

\begin{tabular}{|l|l|l|l|}
\hline & Native & Search & Social \\
\hline Sessions & $238055(53.3 \%)$ & $195786(43.8 \%)$ & $12112(2.7 \%)$ \\
\hline IEUC & 5.2 & 4.4 & 4.2 \\
\hline \multirow{2}{*}{ Browsing } & $55.6 \%:$ article $\rightarrow$ article & $58.8 \%:$ article $\rightarrow$ article & $66.7 \%:$ article $\rightarrow$ article \\
Pattern & $20.6 \%:$ topic page $\rightarrow$ article & $16.9 \%:$ topic page $\rightarrow$ article & $9.1 \%:$ gallery $\rightarrow$ gallery \\
& $5.7 \%:$ sub-topic page $\rightarrow$ article & $7.9 \%:$ sub-topic page $\rightarrow$ article & $4.6 \%:$ article $\rightarrow$ quiz \\
\hline \multirow{2}{*}{ Diversifying } & $73.0 \%:$ topic page $\rightarrow$ topic page & $54.6 \%:$ topic page $\rightarrow$ topic page & $45.1 \%:$ topic page $\rightarrow$ quiz \\
Pattern & $50.8 \%:$ forum $\rightarrow$ sub-topic page & $52.3 \%:$ forum $\rightarrow$ sub-topic page & $42.1 \%:$ article $\rightarrow$ topic page \\
& $50.6 \%:$ forum $\rightarrow$ topic page & $37.7 \%:$ sub-topic page $\rightarrow$ topic page & $26.3 \%:$ article $\rightarrow$ sub-topic page \\
\hline
\end{tabular}

In the literature, a special consideration has be given to sessions where 

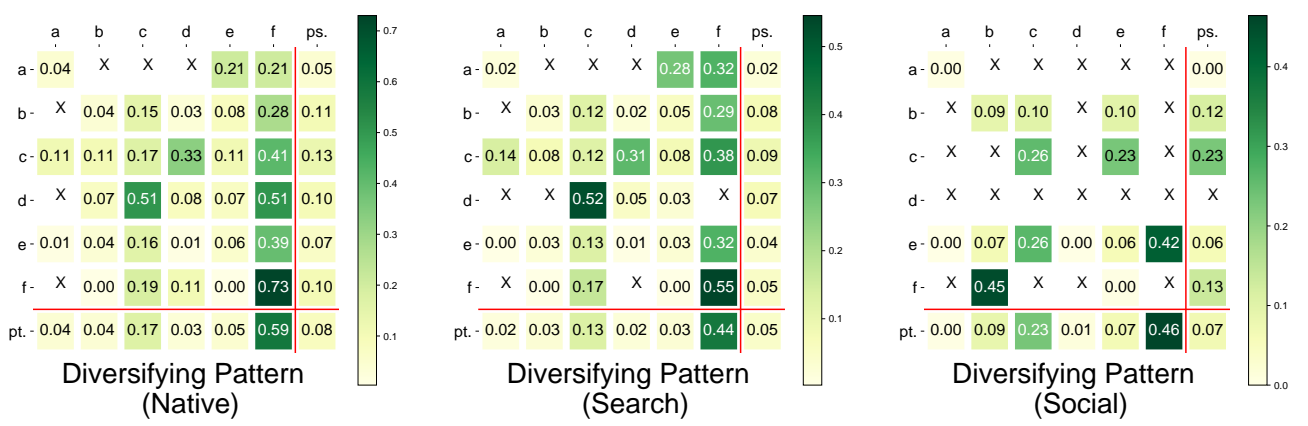

Figure 11: Diversifying Pattern matrix associated with $P_{i \rightarrow j}^{\operatorname{trans}(A)}$ for activity of the sessions with more than 4 requests divided in 3 groups: Native activity (left), Search activity (middle), and Social activity (right).

an external search engine is used to find the website and not a specific content [TASJ14]. These sessions would be identified by the requests from a search engine to the home page of the website. In our study we have ommitted this disctinction, because such sessions represent a rather small part (14.7\% of the Search activity sessions), and no remarkable differences were identified in this group.

\section{Discussion about the structure of the website}

In this section, we relate the observations that we have made throughout this work to the structure of Melty website in order to make educated guesses about the navigation behavior of users and how it is influenced by this structure.

As described in Section 3.1, among the hyperlinks which allow to navigate from page to page, an important part of the links on Melty connect topics to subtopics, subtopics to content pages (articles, forums, ... ) in a tree-like fashion. These structural links, and the underlying page network structure, accounts for a part of the requests constituting browsing paths of users. These are represented in Figure 12 with black arrows.

Other links allow more horizontal navigation, for example links anchored in the text of an article, or dynamical recommendation links. While we cannot make a precise count of every kind of links on any article page from our data, it is clear that a large majority of links on a typical article page 




Figure 12: Schematic representation of the structure of the Melty website. It shows structure-induced, non-diversifying navigation paths (black arrows), and diversifying, cross-topic paths (red arrows).

lead to articles on the same topic and even on the same subtopic. Indeed, the dominating recommender system used on the Melty website (that we cannot describe in details here) favors popularity inside a same topic.

From this description, we can get a better understanding of what we have observed in Section 6. When a session is rather chain-like (as in Cluster A for example), we may assume that the user begins navigation from a topic page, chooses an article, and then follows static links or recommendation links which lead predominantly to other articles of the same topic. It would explain that such sessions are characterized by predominant article to article requests and relatively low diversity. On the other hand, a cluster with higher consumption diversity, like Cluster F, contains longer star-like sessions. In this case, users access articles from a topic page, but also other kinds of pages, possibly changing topic in the process. Diversification probably occurs mostly at the menu level: either users navigate through the menu bar or they use multi-tabbing.

This analysis gives clues to understand how the navigation behavior of users is influenced by the structure of the website. In particular, we conjecture that the scarcity of horizontal links, connecting articles in a given topic to articles in another one, are likely to have a significant impact on the consumption diversity in chain-like sessions. 


\section{Conclusion}

In the course of this study we have addressed the issue of the diversity of browsing paths of sessions over the page network of the informationentertainment website www.melty.fr using server transaction logs. As many other websites, Melty presents a twofold classification of pages, reflecting on the one hand the functional role of a page in the website structure, and on the other hand its type of content. We analyzed three weeks of browsing activity on the website using the server log files. We measured the overall activity in time and grouped the requests in sessions in order to analyze navigation at this scale. Crucially, we evaluated the diversity of the contents consumed by sessions using Shannon entropy. Then, our analysis focused on sessions with more than 4 requests, as it was found to be an appropriate threshold to scrutinize the behavior of users on Melty. It notably showed that sessions originating from social platforms display lower diversity, with larger fluctuations. The measure of aggregated characteristics of the dataset also indicated that pages play a different role in the underlying page network of the website depending on their function category (e.g. topic page or article).

These first results motivated the study of consumed diversity in clusters, based on relevant features of sessions (such as duration or characteristics of the session graphs). These clusters exhibit distinct browsing behaviors, and our analysis revealed that consumption diversity is related to the length and number of requests in a session, but also to the degree to which a session graph is star-like or chain-like. Then, we investigated how the twofold classification of pages is related to these differences in browsing behaviors. Specifically, we measured the functional roles of pages which allow to change topic and thus to diversify consumption. Relating the previous observations to the website design, we could make hypotheses on the likely mechanisms by which users browse the website.

Finally, we made the conjecture that the low-diversity consumption which is observed in chain-like sessions may be related to the rarity of links going from an article to another one with a different topic. This final remark is particularly important considering recommendation links, and it is what can be leveraged most successfully for the improvement of the consumption diversity. The choice of recommending articles on the same topic is a reasonable design choice, as it guarantees a certain degree of accuracy. This means that users are prone to follow such links. However, it also tends to confine the user in a bubble where exploring diverse contents is much less at hand. Of all 
requests made by users following horizontal links between articles, only $4 \%$ of them resulted in a change of topic. This remark is especially relevant in the interaction of bubble-like phenomena between social platforms and website structure. In a social platform, connected users (e.g. friends or followers) can exhibits low diversity in consumption due to homophily, but this lack of diversity can be perpetuated by insufficient availability of diversfying mechanisms in the structure of the websites to which the social browsing leads. In a future work, we consider evaluating experimentally how users react to a greater choice in terms of diversity; in particular, if the website changes the algorithm for recommending related links in article pages, it would be interesting to measure how (and if) the consumed diversity changes with respect to the proposed diversity.

\section{Acknowledgements}

We thank Metly's team, especially Julien Palard for the access to the data. This work has been funded by the French National Agency of Research (ANR) under grant ANR-15-CE38-0001 (Algodiv).

\section{References}

[A+16] Charu C Aggarwal et al. Recommender systems. Springer, 2016.

[ACDN12] Maristella Agosti, Franco Crivellari, and Giorgio Maria Di Nunzio. Web log analysis: a review of a decade of studies about information acquisition, inspection and interpretation of user interaction. Data Mining and Knowledge Discovery, 24(3):663$696,2012$.

[BGST05] Christian Bomhardt, Wolfgang Gaul, and Lars SchmidtThieme. Web robot detection-preprocessing web logfiles for robot detection. In New developments in classification and data analysis, pages 113-124. Springer, 2005.

[BJM83] Lalit R Bahl, Frederick Jelinek, and Robert L Mercer. A maximum likelihood approach to continuous speech recognition. IEEE Transactions on Pattern Analysis 6 Machine Intelligence, (2):179-190, 1983. 
[BJN $\left.{ }^{+} 15\right]$ Pablo Barberá, John T Jost, Jonathan Nagler, Joshua A Tucker, and Richard Bonneau. Tweeting from left to right: Is online political communication more than an echo chamber? Psychological science, 26(10):1531-1542, 2015.

[Bra01] Ulrik Brandes. A faster algorithm for betweenness centrality. Journal of mathematical sociology, 25(2):163-177, 2001.

[BTLG13] Abdelhamid Salah Brahim, Lionel Tabourier, and Bénédicte Le Grand. A data-driven analysis to question epidemic models for citation cascades on the blogosphere. In 7th International AAAI Conference on Weblogs and Social Media, 2013.

[CC01] Hui-Min Chen and Michael D Cooper. Using clustering techniques to detect usage patterns in a web-based information system. Journal of the American Society for Information Science and Technology, 52(11):888-904, 2001.

[CPP00] Ed H Chi, Peter Pirolli, and James Pitkow. The scent of a site: A system for analyzing and predicting information scent, usage, and usability of a web site. In Proceedings of the SIGCHI conference on Human Factors in Computing Systems, pages 161-168. ACM, 2000.

[CR13] Min Chen and Young U Ryu. Facilitating effective user navigation through website structure improvement. IEEE Transactions on Knowledge and Data Engineering, 25(3):571-588, 2013.

[DG11] Derek Doran and Swapna S Gokhale. Web robot detection techniques: overview and limitations. Data Mining and Knowledge Discovery, 22(1-2):183-210, 2011.

[DSLS17] Dimitar Dimitrov, Philipp Singer, Florian Lemmerich, and Markus Strohmaier. What makes a link successful on wikipedia? In Proceedings of the 26th International Conference on World Wide Web, pages 917-926. International World Wide Web Conferences Steering Committee, 2017.

[EMC10] Nathan Eagle, Michael Macy, and Rob Claxton. Network diversity and economic development. Science, 328(5981):1029-1031, 2010. 
[FGR16] Seth Flaxman, Sharad Goel, and Justin M Rao. Filter bubbles, echo chambers, and online news consumption. Public opinion quarterly, 80(S1):298-320, 2016.

[FH09] Daniel Fleder and Kartik Hosanagar. Blockbuster culture's next rise or fall: The impact of recommender systems on sales diversity. Management science, 55(5):697-712, 2009.

[FSCJ02] Yongjian Fu, Ming-Yi Shih, Mario Creado, and Chunhua Ju. Reorganizing web sites based on user access patterns. Intelligent Systems in Accounting, Finance 83 Management, 11(1):39-53, 2002.

[GGTN17] Joris Guérin, Olivier Gibaru, Stéphane Thiery, and Eric Nyiri. Clustering for different scales of measurement-the gap-ratio weighted k-means algorithm. arXiv preprint arXiv:1703.07625, 2017.

[HKK $\left.{ }^{+} 15\right]$ Aaron Halfaker, Oliver Keyes, Daniel Kluver, Jacob ThebaultSpieker, Tien Nguyen, Kenneth Shores, Anuradha Uduwage, and Morten Warncke-Wang. User session identification based on strong regularities in inter-activity time. In Proceedings of the 24th International Conference on World Wide Web, pages 410-418, 2015.

[HSGS13] Denis Helic, Markus Strohmaier, Michael Granitzer, and Reinhold Scherer. Models of human navigation in information networks based on decentralized search. In Proceedings of the 24th Conference on Hypertext and Social Media, pages 89-98. ACM, 2013.

[Jan08] Bernard J Jansen. Handbook of research on web log analysis. IGI Global, 2008.

[JK08] Rosie Jones and Kristina Lisa Klinkner. Beyond the session timeout: automatic hierarchical segmentation of search topics in query logs. In Proceedings of the 17th Conference on Information and Knowledge Management, pages 699-708. ACM, 2008. 
[KZN $\left.{ }^{+} 15\right]$ Juhi Kulshrestha, Muhammad Bilal Zafar, Lisette Espin Noboa, Krishna P Gummadi, and Saptarshi Ghosh. Characterizing information diets of social media users. In 9th International AAAI Conference on Web and Social Media, 2015.

[LCB16] Amaury L'Huillier, Sylvain Castagnos, and Anne Boyer. Modéliser la diversité au cours du temps pour détecter le contexte dans un service de musique en ligne. Revue des Sciences et Technologies de l'Information, 2016.

[LK07] Haibin Liu and Vlado Kešelj. Combined mining of web server $\operatorname{logs}$ and web contents for classifying user navigation patterns and predicting users' future requests. Data $\& 3$ Knowledge Engineering, 61(2):304-330, 2007.

[McA89] Ray McAleese. Navigation and browsing in hypertext. Hypertext: theory into practice, pages 6-44, 1989.

[MF01] Alan L Montgomery and Christos Faloutsos. Identifying web browsing trends and patterns. Computer, 34(7):94-95, 2001.

[MS98] Sharon McDonald and Rosemary J Stevenson. Navigation in hyperspace: An evaluation of the effects of navigational tools and subject matter expertise on browsing and information retrieval in hypertext. Interacting with computers, 10(2):129-142, 1998.

$\left[\mathrm{NDBB}^{+} 19\right]$ Adrien Nouvellet, Florence D'Alché-Buc, Valérie Baudouin, Christophe Prieur, and François Roueff. Discovery of usage patterns in digital library web logs using Markov modeling. Preprint, 2019.

[NHH $\left.{ }^{+} 14\right]$ Tien T Nguyen, Pik-Mai Hui, F Maxwell Harper, Loren Terveen, and Joseph A Konstan. Exploring the filter bubble: the effect of using recommender systems on content diversity. In Proceedings of the 23rd international conference on World wide web, pages 677-686. ACM, 2014.

[NHJ08] David Nicholas, Paul Huntington, and Hamid R Jamali. User diversity: as demonstrated by deep log analysis. The Electronic Library, 26(1):21-38, 2008. 
[NHW05] David Nicholas, Paul Huntington, and Anthony Watkinson. Scholarly journal usage: the results of deep log analysis. Journal of documentation, 61(2):248-280, 2005.

[NOFM15] Dimitar Nikolov, Diego FM Oliveira, Alessandro Flammini, and Filippo Menczer. Measuring online social bubbles. PeerJ Computer Science, 1:e38, 2015.

[OCJ12] Lukasz Olejnik, Claude Castelluccia, and Artur Janc. Why johnny can't browse in peace: On the uniqueness of web browsing history patterns. In 5th Workshop on Hot Topics in Privacy Enhancing Technologies (HotPETs 2012), 2012.

[Par11] Eli Pariser. The filter bubble: What the Internet is hiding from you. Penguin UK, 2011.

[Pet93] Thomas A Peters. The history and development of transaction log analysis. Library hi tech, 11(2):41-66, 1993.

[PSL13] Huy Pham, Cyrus Shahabi, and Yan Liu. Ebm: an entropybased model to infer social strength from spatiotemporal data. In Proceedings of the 2013 ACM SIGMOD International Conference on Management of Data, pages 265-276. ACM, 2013.

[PWZL16] Ashwin Paranjape, Robert West, Leila Zia, and Jure Leskovec. Improving website hyperlink structure using server logs. In Proceedings of the Ninth ACM International Conference on Web Search and Data Mining, pages 615-624. ACM, 2016.

[RRS15] Francesco Ricci, Lior Rokach, and Bracha Shapira. Recommender systems: introduction and challenges. In Recommender systems handbook, pages 1-34. Springer, 2015.

[SMHM99] Craig Silverstein, Hannes Marais, Monika Henzinger, and Michael Moricz. Analysis of a very large web search engine query log. In ACM SIGIR Forum, volume 33, pages 6-12. ACM, 1999.

[SPWL14] Aju Thalappillil Scaria, Rose Marie Philip, Robert West, and Jure Leskovec. The last click: Why users give up information network navigation. In Proceedings of the 7th ICWSDM Conference, pages 213-222. ACM, 2014. 
[Ste08] Dick Stenmark. Identifying clusters of user behavior in intranet search engine log files. Journal of the American Society for Information Science and Technology, 59(14):2232-2243, 2008.

[Sti98] Andrew Stirling. On the economics and analysis of diversity. Science Policy Research Unit (SPRU), Electronic Working Papers Series, Paper, 28:1-156, 1998.

[Sti07] Andy Stirling. A general framework for analysing diversity in science, technology and society. Journal of the Royal Society Interface, 4(15):707-719, 2007.

[SY01] Ramakrishnan Srikant and Yinghui Yang. Mining web logs to improve website organization. $W W W, 1: 430-437,2001$.

[SZDV $\left.{ }^{+} 17\right]$ Ana Lucía Schmidt, Fabiana Zollo, Michela Del Vicario, Alessandro Bessi, Antonio Scala, Guido Caldarelli, H Eugene Stanley, and Walter Quattrociocchi. Anatomy of news consumption on facebook. Proceedings of the National Academy of Sciences, 114(12):3035-3039, 2017.

[TASJ14] Michele Trevisiol, Luca Maria Aiello, Rossano Schifanella, and Alejandro Jaimes. Cold-start news recommendation with domain-dependent browse graph. In Proceedings of the 8th ACM Conference on Recommender systems, pages 81-88. ACM, 2014.

[TK04] Pang-Ning Tan and Vipin Kumar. Discovery of web robot sessions based on their navigational patterns. In Intelligent Technologies for Information Analysis, pages 193-222. Springer, 2004.

[VC11] Saúl Vargas and Pablo Castells. Rank and relevance in novelty and diversity metrics for recommender systems. In Proceedings of the fifth ACM conference on Recommender systems, pages 109-116. ACM, 2011.

[WC16] Simon Wakeling and Paul Clough. Determining the optimal session interval for transaction log analysis of an online library catalogue. In European Conference on Information Retrieval, pages 703-708. Springer, 2016. 
[WL12] Robert West and Jure Leskovec. Human wayfinding in information networks. In Proceedings of the 21st international conference on World Wide Web, pages 619-628. ACM, 2012.

[Wol08] Dietmar Wolfram. Search characteristics in different types of web-based ir environments: Are they the same? Information processing \&3 management, 44(3):1279-1292, 2008.

$\left[Z_{K L}^{+} 10\right]$ Tao Zhou, Zoltán Kuscsik, Jian-Guo Liu, Matúš Medo, Joseph Rushton Wakeling, and Yi-Cheng Zhang. Solving the apparent diversity-accuracy dilemma of recommender systems. PNAS, 107(10):4511-4515, 2010. 